\title{
Electromagnetic Field Coupling to Transmission Lines: A Model for the Risers
}

\author{
Gaspard Lugrin, Nicolas Mora and Farhad Rachidi \\ EMC Laboratory \\ École polytechnique fédérale de Lausanne (EPFL) \\ Lausanne, Switzerland \\ gaspard.lugrin@epfl.ch
}

\author{
Sergey Tkachenko \\ Lehrstuhl für EMV \\ Otto-von-Guericke-Universität (OVGU) \\ Magdeburg, Germany
}

\begin{abstract}
In this paper, an improved model of the vertical risers of a transmission line is proposed and validated. The proposed improved model, which can be readily implemented in the frame of a classical transmission line formulation, provides accurate results at frequencies much higher than the upper frequency limit of the classical transmission line theory. For example, for the assessed line (with a height above the ground of $10 \mathrm{~cm}$ ), the improved model is still fairly accurate at around $1 \mathrm{GHz}$, whereas the classical model starts to deviate from the accurate solution at around $150 \mathrm{MHz}$.
\end{abstract}

Keywords - Baum-Liu-Tesche (BLT); transmission line (TL); radiation; vertical risers.

\section{INTRODUCTION}

In the classical transmission line (TL) theory, the effect of vertical risers should be appropriately taken into account when calculating the response of a line to either a lumped source or an exciting electromagnetic field. Generally, vertical risers are modeled using lumped elements [1]. An exact modelling of a line with vertical risers is a rather complicated full-wave problem that goes beyond the TL theory and is currently under study (see e.g., [2]-[6]).

In the framework of the TL theory, several attempts were made in order to model the risers more accurately than using lumped elements, and therefore extend the validity range of the theory. Vance [7] modeled the risers as a conical transmission line with its upper end terminated in its characteristic impedance. Hence, the effect of the risers is essentially an increase in the effective line length by the length of the risers. The field coupling to the risers was then modelled as distributed sources along the risers and an expression for the current at the extremities of the horizontal line was given.

More recently, Pignari and Bellan [8] presented a model in which the risers were represented as cylindrical transmission lines. In this case, the characteristic impedance is not the same as the impedance of the horizontal part of the line. Hence they model the system as three cascaded lines and excited by distributed sources.

Chabane et al. [9] considered the case of a line is excited by a lumped source at one extremity and proposed to include a distributed resistance along the line to simulate the losses due to radiation effect. However, radiation occurs mainly around discontinuities of the lines, such as risers or bends, and no radiation is generated by the main transverse electromagnetic (TEM) mode propagating along the straight, central part of the line $[10]$.

In this paper, we present an improved model for the vertical risers of a line, within the framework of the TL theory. The proposed model uses a distributed-source representation of the coupling to risers, similar to Pignari and Bellan [8], instead of a lumped source representation as in the original Agrawal et al. model [11]. Furthermore, the proposed model includes a resistance accounting for high frequency radiation effects.

We consider the case of a lossless single-wire line above a perfectly-conducting ground excited by a plane wave. In a real scenario, ground and dielectric losses will attenuate and distort the propagation of the induced signals. The accuracy of the proposed model is validated by comparison with a full-wave numerical model in the frequency domain.

\section{MODELING}

A schematic diagram of the assessed system is presented in Fig. 1. A perfect electric conductor (PEC) wire of circular cross-section with diameter $2 a$ and length $L$ is located at a height $h$ above a perfect ground plane. The wire is terminated to ground with two lumped impedances $Z_{1}$ and $Z_{2}$. The line is illuminated with a plane wave, characterized by its amplitude $E_{0}$ and three angles: namely, the elevation angle $\psi$, the azimuth angle $\varphi$, and the polarization angle $\alpha$ of the electric field [12].

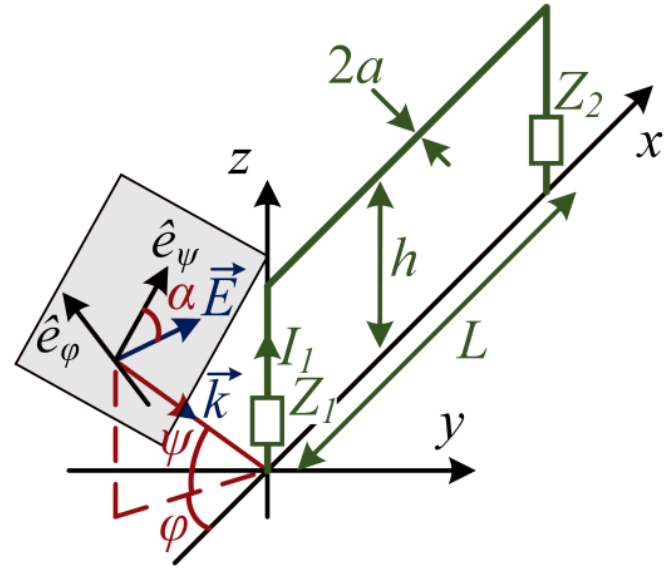

Fig. 1. Schematic diagram of the assessed system (adapted from [12]).

The BLT equation describing the induced currents at the line terminals $I_{1}$ and $I_{2}$ can be written as [12]:

$$
\left[\begin{array}{l}
I_{1} \\
I_{2}
\end{array}\right]=\frac{1}{Z_{c}}\left[\begin{array}{cc}
1-\rho_{1} & 0 \\
0 & 1-\rho_{2}
\end{array}\right]\left[\begin{array}{cc}
-\rho_{1} & e^{\gamma L} \\
e^{\gamma L} & -\rho_{2}
\end{array}\right]^{-1}\left[\begin{array}{l}
S_{1} \\
S_{2}
\end{array}\right]
$$

where $Z_{C}$ is the line characteristic impedance, $\rho_{1}$ and $\rho_{2}$ are the reflection coefficients at the left and right terminal respectively, and $\gamma=\mathrm{j} k$ is the propagation constant along the line. $S_{1}$ and $S_{2}$ are source terms which will be defined in the following sections. 


\section{A. Basic model: Approximate Solution for Lumped Sources}

In [12], expressions of the source terms are given under the assumption that the vertical variation of the field along the risers can be neglected:

$$
\begin{aligned}
& S_{1}=E_{0}\left(\mathrm{e}^{\left(\gamma-\mathrm{j} k_{x}\right) L}-1\right) \mathrm{j} k_{z} h\left(\frac{A}{\gamma-\mathrm{j} k_{x}}-\frac{B}{\mathrm{j} k_{z}}\right) \\
& S_{2}=-E_{0} \mathrm{e}^{\gamma L}\left(\mathrm{e}^{-\left(\gamma+\mathrm{j} k_{x}\right) L}-1\right) \mathrm{j} k_{z} h\left(\frac{A}{-\left(\gamma+\mathrm{j} k_{x}\right)}-\frac{B}{\mathrm{j} k_{z}}\right)
\end{aligned}
$$

with

$$
\begin{array}{ll}
A=\cos \alpha \sin \psi \cos \varphi+\sin \alpha \sin \varphi & k_{x}=k \cos \psi \cos \varphi \\
B=\cos \alpha \cos \psi & k_{z}=k \sin \psi
\end{array}
$$

This model is expected to be accurate at low frequencies, when the length of the riser is electrically short, that is under the classical transmission line assumptions.

\section{B. Exact-field model: Exact Solution for the Lumped Sources}

If the field variation along the vertical risers are taken into account to compute the lumped source associated with the vertical risers, the source terms are given by the following expressions:

$$
\begin{aligned}
& S_{1}=\frac{E_{0}}{2}\left(\mathrm{e}^{\left(\gamma-j k_{x}\right) L}-1\right)\left(\mathrm{e}^{\mathrm{j} k_{z} h}-\mathrm{e}^{-\mathrm{j} k_{z} h}\right)\left(\frac{A}{\gamma-j k_{x}}-\frac{B}{\mathrm{j} k_{z}}\right) \\
& S_{2}=-\frac{E_{0}}{2} \mathrm{e}^{\gamma \mathrm{L}}\left(\mathrm{e}^{-\left(\gamma+j k_{x}\right) L}-1\right)\left(\mathrm{e}^{\mathrm{j} k_{z} h}-\mathrm{e}^{-\mathrm{j} k_{z} h}\right)\left(\frac{A}{-\left(\gamma+j k_{x}\right)}-\frac{B}{\mathrm{j} k_{z}}\right)
\end{aligned}
$$

This model is expected to provide more accurate results than the basic model at high-frequency.

\section{Distributed-source model}

In this model, the line is represented as three cascaded transmission lines, corresponding respectively to the left riser, the horizontal part and the right riser. As shown in Fig. 2, the lines corresponding to the risers are excited by distributed sources whose amplitude corresponds to the vertical component of the electric field, whereas the horizontal line is excited by the horizontal component of the electric field (within the Agrawal et al. model for field-to-wire coupling [11].

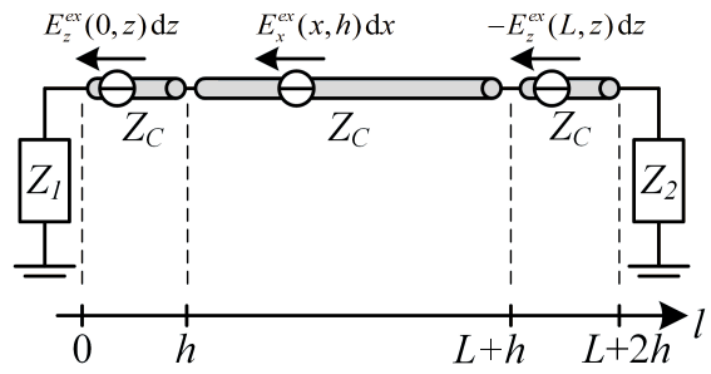

Fig. 2. Modelling of a TL with distributed sources along the risers.

As the three lines in Fig. 2 are supposed to have the same characteristic impedance, they can be merged into a single line of length $L+2 h$. This simplifies the computations and allows the use of the standard BLT equation. In this model, $L$ is replaced by $L+2 h$ in (1) and the following expressions for the source terms can be readily derived |7th Asia Pacific International Symposium on Electromagnetic Compatibility |

$$
\begin{aligned}
& S_{1}=\frac{E_{0}}{2}\left[B\left(\frac{\mathrm{e}^{j\left(k+k_{z}\right) h}-1}{j\left(k+k_{z}\right)}+\frac{\mathrm{e}^{j\left(k-k_{z}\right) h}-1}{j\left(k-k_{z}\right)}\right)+C \mathrm{e}^{j k h}\left(\frac{\mathrm{e}^{j\left(k-k_{x}\right) L}-1}{j\left(k-k_{x}\right)}\right)\right. \\
& \left.-B \mathrm{e}^{-j k_{x} L} \mathrm{e}^{j k(L+h)}\left(\mathrm{e}^{j k_{z} h} \frac{\mathrm{e}^{j\left(k-k_{z}\right) h}-1}{j\left(k-k_{z}\right)}+\mathrm{e}^{-j k_{z} h} \frac{\mathrm{e}^{j\left(k+k_{z}\right) h}-1}{j\left(k+k_{z}\right)}\right)\right] \\
& S_{2}=-\frac{E_{0}}{2} \mathrm{e}^{j k(L+2 h)}\left[B\left(\frac{\mathrm{e}^{-j\left(k-k_{z}\right) h}-1}{-j\left(k-k_{z}\right)}+\frac{\mathrm{e}^{-j\left(k+k_{z}\right) h}-1}{-j\left(k+k_{z}\right)}\right)\right. \\
& +C \mathrm{e}^{-j k h}\left(\frac{\mathrm{e}^{-j\left(k+k_{x}\right) L}-1}{-j\left(k+k_{x}\right)}\right) \\
& \left.-B \mathrm{e}^{-j k_{x} L} \mathrm{e}^{-j k(L+h)}\left(\mathrm{e}^{j k_{z} h} \frac{\mathrm{e}^{-j\left(k+k_{z}\right) h}-1}{-j\left(k+k_{z}\right)}+\mathrm{e}^{-j k_{z} h} \frac{\mathrm{e}^{-j\left(k-k_{z}\right) h}-1}{-j\left(k-k_{z}\right)}\right)\right]
\end{aligned}
$$

with $C=A\left(\mathrm{e}^{j k_{z} h}-\mathrm{e}^{-j k_{z} h}\right)$.

Note that this model is similar to Pignari and Bellan's [8], with the exception that the characteristic impedance of the risers is supposed here to be the same as that of the horizontal line. This model is expected to provide more accurate solutions than the basic and exact-field models, but may overestimate the amplitude of the induced current, since it does not consider the radiation effects.

\section{Proposed model}

The distributed-source model (Section II.C) do not consider the radiation effects associated with the risers, which may become significant when the wavelength of the induced current is in the same order of magnitude as the length of the riser.

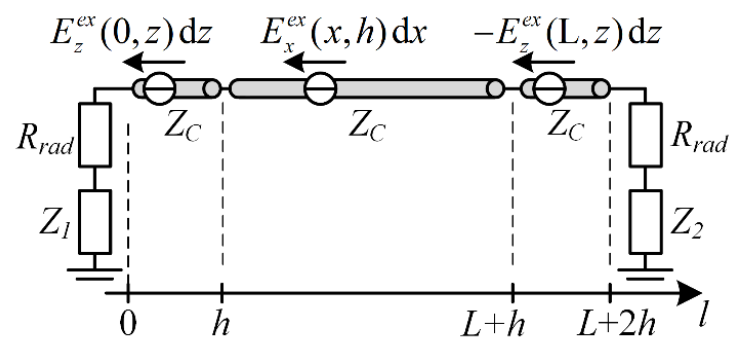

Fig. 3. Modelling of a transmission line with radiation resistances.

As shown in Fig. 3, in order to include this radiation effect, an additional resistance, corresponding to the radiation resistance of an electrically very short monopole of length $h$, adapted from the expression for a dipole [13], is added to the terminal:

$$
R_{\text {rad }}=160 \pi^{2}\left(\frac{h}{\lambda}\right)^{2}
$$

where $\lambda=c / f$ is the wavelength. In other words, the impedance at the left (respectively right) terminal is considered to be constituted of the actual impedance at the left (respectively right) terminal plus the radiation resistance. Hence, the corresponding reflection coefficients can be obtained as:

$$
\rho_{i}=\frac{\left(Z_{i}+R_{\text {rad }}\right)-Z_{C}}{\left(Z_{i}+R_{\text {rad }}\right)+Z_{C}} \quad i=1 ; 2
$$

This model can be simulated in a straightforward way by inserting the source terms (5) into the BLT equations (1), in which the length of the line $L$ have been replaced by $L+2 h$, and 
where the reflection coefficients $\rho_{1}$ and $\rho_{2}$ have been modified to consider the radiation resistance.

This model is expected to be more accurate than the previous ones at frequencies beyond the limit of the TL theory.

\section{VALIDATION}

A summary of the parameters of the TL used for the validation is shown in Table I.

TABLE I. PARAMETERS OF THE TRANSMISSION LINE

\begin{tabular}{ccc}
\hline \hline Parameter & Value & Units \\
\hline \hline Length $L$ & 3 & $\mathrm{~m}$ \\
\hline Radius $a$ & 0.7 & $\mathrm{~mm}$ \\
\hline Height $h$ & 10 & $\mathrm{~cm}$ \\
\hline Impedance $Z_{I}$ & 339 & $\Omega$ \\
\hline Impedance $Z_{2}$ & 0 & $\Omega$ \\
\hline Elevation angle $\psi$ & 45 & $\circ$ \\
\hline Azimuth angle $\varphi$ & 0 & $\circ$ \\
\hline Polarization angle $\alpha$ & 0 & $\circ$
\end{tabular}

The transfer function between the incident plane wave and the current induced in the first terminal at the level of the ground is shown in Fig. 4. Results obtained using the considered TL models for the risers are compared with fullwave results using CST Microwave Studio.

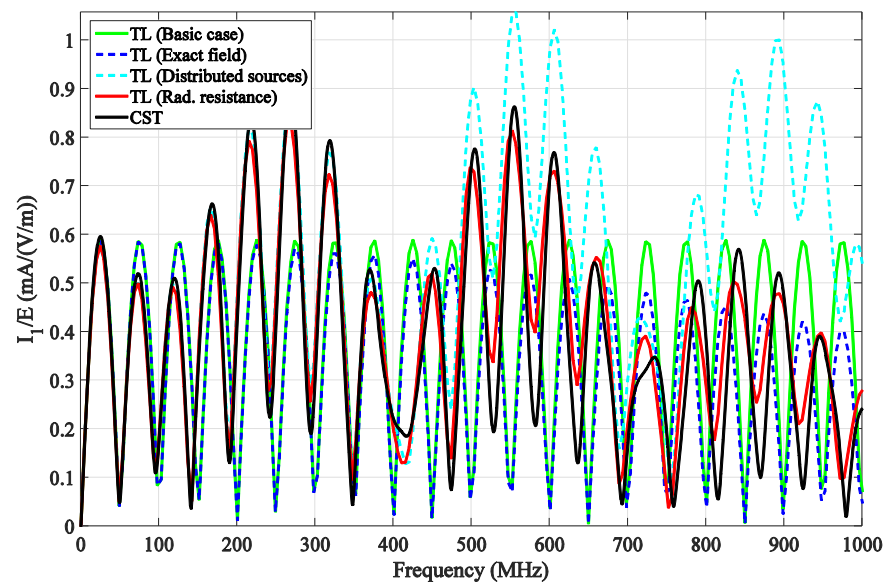

Fig. 4. $I_{1} / E_{0}$ transfer functions calculated with four models based on the classical TL theory (basic case, exact field, distributed sources and radiation resistances) and with a full-wave code (CST).

It can be seen from Fig. 4 that all the considered models provide basically the same results at low frequencies up to about $60 \mathrm{MHz}$ (the TL theory is supposed to be valid up to about $150 \mathrm{MHz}$ here). The basic model and the exact-field model provide accurate results up to about $100 \mathrm{MHz}$. The distributed source model for the risers provides very accurate results up to about $400 \mathrm{MHz}$, but deviates significantly at higher frequencies with respect to full-wave results.

On the other hand, the proposed model for the risers provides results with reasonable accuracy up to about $1 \mathrm{GHz}$, well beyond the classical limit of the TL theory.
| 7th Asia Pacific International Symposium on Electromagnetic Compatibility

\section{CONCLUSION}

In this paper, an improved model of the vertical risers was proposed and validated by comparison with full-wave simulations. The proposed model, which can be readily implemented in the frame of a classical transmission line formulation, provides accurate results at frequencies much higher than the upper frequency limit of the classical transmission line theory. For example, for the assessed line (with a height above the ground of $10 \mathrm{~cm}$ ), the improved model is still fairly accurate up to $1 \mathrm{GHz}$, whereas the classical model is no longer able to provide accurate results for frequencies higher than about $150 \mathrm{MHz}$.

\section{ACKNOWLEDGMENT}

This work was financed by the Armasuisse Science and technology (Contract Nr. 8003508747).

\section{REFERENCES}

[1] F. Rachidi, "A Review of Field-to-Transmission Line Coupling Models with Special Emphasis to Lightning-Induced Voltages", IEEE Transactions on Electromagnetic Compatibility, Vol 54, No. 4, pp. 898 $-911,2012$.

[2] J. Nitsch and S. Tkachenko, "High-Frequency Multiconductor Transmission-Line Theory," Found Phys, March 2010.

[3] M. Brignone, F. Delfino, R. Procopio, M. Rossi, F. Rachidi, and S. Tkachenko, "An effective approach for high-frequency electromagnetic field-to-line coupling analysis based on regularization techniques," Electromagnetic Compatibility, IEEE Transactions on, vol. 54, no. 6, pp. 1289-1297, Dec 2012.

[4] S. Tkachenko, F. Middelstaedt, J. Nitsch, R. Vick, G. Lugrin, and F. Rachidi, "High-Frequency Electromagnetic Field Coupling to a Long Finite Line with Vertical Risers," in URSI, 2014.

[5] G. Lugrin, S. Tkachenko, F. Rachidi, M. Rubinstein, and R. Cherkaoui, "High-frequency electromagnetic coupling to multiconductor transmission lines of finite length," Electromagnetic Compatibility, IEEE Transactions on, vol. PP, no. 99, pp. 1-10, 2015.

[6] R. Rambousky, J. Nitsch, and S. Tkachenko, "Application of transmission-line super theory to classical transmission lines with risers," Advances in Radio Science, vol. 13, pp. 161-168, 2015.

[7] E. Vance, Coupling to Shielded Cables. New-York: John Wiley \& Sons, 1978.

[8] S. Pignari and D. Bellan, "Incorporating vertical risers in the transmission line equations with external sources," in International Symposium on Electromagnetic Compatibility (EMC 2004), vol. 3, Aug 2004, pp. 974-979.

[9] S. Chabane, P. Besnier, and M. Klingler, "Enhanced transmission line theory: Frequencydependent line parameters and their insertion in a classical transmission line equation solver," in EMC EUROPE 2013, pp. 326-331.

[10] F. Rachidi and S. Tkachenko, Eds., Electromagnetic Field Interaction with Transmission Lines. From Classical Theory to HF Radiation Effects. Southampton, Boston: WITpress, 2008.

[11] A. Agrawal, H. Price, and S. Gurbaxani, "Transient Response of Multiconductor Transmission Lines Excited by a Nonuniform Electromagnetic Field," IEEE Transactions on EMC, vol. 22, pp. 119 129, May 1980.

[12] F. Tesche, M. Ianoz, and T. Karlsson, EMC analysis methods and computational models. New York: John Wiley \& Sons, 1997.

[13] C. A. Balanis, Antenna Theory: Analysis and Design. Wiley, 2005. 